\title{
ASYMPTOTIC PROPERTIES OF A LEADER ELECTION ALGORITHM
}

\author{
RAVI KALPATHY *** AND \\ HOSAM M. MAHMOUD, ${ }^{* * * *}$ The George Washington University \\ MARK DANIEL WARD, ${ }^{* * * *}$ Purdue University
}

\begin{abstract}
We consider a serialized coin-tossing leader election algorithm that proceeds in rounds until a winner is chosen, or all contestants are eliminated. The analysis allows for either biased or fair coins. We find the exact distribution for the duration of any fixed contestant; asymptotically, it turns out to be a geometric distribution. Rice's method (an analytic technique) shows that the moments of the duration contain oscillations, which we give explicitly for the mean and variance. We also use convergence in the Wasserstein metric space to show that the distribution of the total number of coin flips (among all participants), suitably normalized, approaches a normal limiting random variable.
\end{abstract}

Keywords: Leader election; analytic combinatorics; Rice's method; fixed point; contraction method; Wasserstein metric space; weak convergence

2010 Mathematics Subject Classification: Primary 60C05

Secondary 05C05; 60F05; 68W40

\section{Introduction}

Leader election is a problem of commonplace use and numerous applications, from scenarios to elect a winner of a contest, to security systems in the case of failure of the existing central coordinator. In this paper we analyze a randomized leader election algorithm that operates by flipping coins to select a winner, with the possibility of no winners at all, as happens sometimes, e.g. in book awards.

At the start we have $n$ contestants, of which ultimately one or none is declared the winner after a fair competition. The contestants go through elimination rounds in which they flip a (possibly biased) coin. Throughout, $p \in(0,1)$ denotes the probability that the coin lands as a head, and $q=1-p$ denotes the probability that the coin lands as a tail. At the first round, those who flip tails are eliminated from the competition, and those who flip heads stay to compete in further rounds. This elimination process goes on until either one contestant is declared a winner, or all contestants are eliminated.

The possibly winnerless algorithm is a variation of an algorithm discussed in [3] and [12], the difference being when all the contestants flip tails in the possibly winnerless algorithms, they get eliminated, whereas in [3] and [12] the contest is reset and starts afresh with all the

\footnotetext{
Received 27 May 2010; revision received 2 February 2011.

* Postal address: Department of Statistics, The George Washington University, 2140 Pennsylvania Avenue, Washington, DC 20052, USA.

** Email address: kalpathy@gwu.edu

*** Email address: hosam@gwu.edu

**** Postal address: Department of Statistics, Purdue University, 150 North University Street, West Lafayette, IN 47907-1451, USA. Email address: mdw@purdue.edu

Research supported by NSF Science \& Technology Center grant CCF-0939370.
} 
contestants reentering the competition again. The variation where all contestants are eliminated presents a realistic assumption. It sometimes happens in real-life competition that an award is not given. For instance, in 1949 the Swedish Academy withheld the Nobel Prize in Literature, when it got deadlocked over four remaining candidates. The two algorithms are related. In fact, the possibly winnerless version provides a benchmark for the other algorithm. The duration of contestants, the number of coin flips, the duration of the entire algorithm, etc. in the possibly winnerless version are stochastically dominated by their counterparts in the other version, and, hence, provide lower bounds for averages.

In the analysis of several variants of leader election algorithms, the typical parameter of interest is the number of rounds till termination (see [3], [5], and [12]). More recent research has branched out to consider other parameters and properties. For instance, in [7] and [8] a version of leader election algorithms is considered, where a demon randomly eliminates some contestants, and the algorithm stops, if all contestants flip tails and are declared winners (survivors) of the competition. Another important parameter is the total number of coin flips; Prodinger [12] found the exact average number of coin flips for a certain leader election algorithm that flips unbiased coins. This parameter is relevant in the serialized version of this class of algorithms, where only one coin is available and is passed from one contestant to the next.

We study the prospects of a contestant, as represented by the distribution of the number of rounds he/she stays, and the speed of the serialized version of the algorithm, as represented by the distribution of the total number of coin flips.

The paper is organized as follows. In Section 2 the exact and asymptotic distributions of the duration of a contestant are derived, and the oscillatory behavior of the mean and variance is given. In Section 3 we obtain the limiting distribution of the total number of flips using convergence of distribution functions in the Wasserstein metric space.

\section{Duration of a contestant}

Let $D_{n, j}$ be the number of rounds that the $j$ th contestant survives when $n$ participants are present at the start. Since the contestants behave stochastically in the same way, $D_{n, j} \stackrel{\mathrm{D}}{=} D_{n, 1}$, where ' $=$, denotes (exact) equality in distribution. So we shall develop results for $D_{n}:=D_{n, 1}$ and drop the second subscript to keep the notation simple. Equivalently, $D_{n}$ is the duration of a randomly selected contestant. For $n=0$ or $n=1$, no election is necessary, so we define $D_{0}=D_{1}=0$.

Theorem 1. The probability mass function for $D_{n}$ is exactly

$$
\mathrm{P}\left(D_{n}=k\right)=p^{k-1}\left(q-\left(1-p^{k-1}\right)^{n-1}+p\left(1-p^{k}\right)^{n-1}\right),
$$

for $n \geq 2$ and $k \geq 1$.

Proof. The duration of the first contestant is $k$ in one of two ways: he/she either loses in $k$ rounds (event $\mathcal{L}_{k}$ ), or wins in $k$ rounds (event $\mathfrak{W}_{k}$ ). The event $\mathscr{L}_{k}$ occurs if he/she flips $k-1$ heads followed by a tail in the $k$ th flip, provided that at least one other contestant flips $k-1$ heads (otherwise, the contest would have stopped sooner). Thus,

$$
\mathrm{P}\left(\mathcal{L}_{k}\right)=p^{k-1} q\left(1-\left(1-p^{k-1}\right)^{n-1}\right) .
$$

The event $\mathfrak{W}_{k}$ occurs if the first contestant initially has $k$ heads, provided that none of the other contestants begins with $k$ heads, but at least one of them begins with $k-1$ heads (otherwise, 
the winning contestant would have won sooner). Thus,

$$
\mathrm{P}\left(\mathcal{W}_{k}\right)=p^{k}\left(\left(1-p^{k}\right)^{n-1}-\left(1-p^{k-1}\right)^{n-1}\right) .
$$

Whence, $\mathrm{P}\left(D_{n}=k\right)=\mathrm{P}\left(\mathcal{L}_{k}\right)+\mathrm{P}\left(\mathfrak{W}_{k}\right)$, and the theorem follows.

In what follows, $\operatorname{Geo}(q)$ stands for a geometric random variable arising from the number of independent, identically distributed trials until the first success, each having probability of success $q$. The notation ' $\stackrel{\text { D }}{\rightarrow}$ ' will denote weak convergence (convergence in distribution), ' $\stackrel{\text { P }}{\rightarrow}$ ' will denote convergence in probability, and ' $\stackrel{\text { a.s. }}{\longrightarrow}$, will denote convergence almost surely.

Corollary 1. As $n \rightarrow \infty$,

$$
D_{n} \stackrel{\mathrm{D}}{\rightarrow} \operatorname{Geo}(q) .
$$

Indeed, not only does $D_{n}$ converge in distribution to $\operatorname{Geo}(q)$, but each of the moments of $D_{n}$ converges to the analogous moment of a $\operatorname{Geo}(q)$ random variable. (We prove this only for the mean; we sketch a proof for the variance. The same analytic methodology yields proofs for higher moments of $D_{n}$, although for conciseness, we do not present such proofs here.) What is interesting here is the asymptotic oscillations that ultimately disappear. We illustrate this disappearance for the mean and variance.

Corollary 2. As $n \rightarrow \infty$,

$$
\begin{gathered}
\mathrm{E}\left[D_{n}\right]=\frac{1}{q}+\left(\frac{1}{\ln p} \sum_{k=-\infty}^{\infty} \Gamma\left(1-\frac{2 \pi \mathrm{i} k}{\ln p}\right) \mathrm{e}^{-2 \pi \mathrm{i} k \log _{1 / p} n}\right) \frac{1}{n}+O\left(\frac{1}{n^{2}}\right), \\
\operatorname{var}\left[D_{n}\right]=\frac{p}{q^{2}}-\left(\frac{2}{(\ln p)^{2}} \sum_{k=-\infty}^{\infty} \Gamma\left(1-\frac{2 \pi \mathrm{i} k}{\ln p}\right) \mathrm{e}^{-2 \pi \mathrm{i} k \log _{1 / p} n}\right) \frac{\ln n}{n}+O\left(\frac{1}{n}\right) .
\end{gathered}
$$

The error terms include small oscillations.

Proof. We use the exact form of the distribution of $D_{n}$ in Theorem 1 as a starting point. This yields the exact mean for $n \geq 2$ :

$$
\mathrm{E}\left[D_{n}\right]=\sum_{k=0}^{\infty} k \mathrm{P}\left(D_{n}=k\right)=\frac{1}{q}-\sum_{k=0}^{\infty} k p^{k-1}\left(1-p^{k-1}\right)^{n-1}+\sum_{k=0}^{\infty} k p^{k}\left(1-p^{k}\right)^{n-1} .
$$

After shifting the index of $k$ in the first sum, we have $\mathrm{E}\left[D_{n}\right]=1 / q-\sum_{k=0}^{\infty} p^{k}\left(1-p^{k}\right)^{n-1}$. Using the binomial expansion $\left(1-p^{k}\right)^{n-1}=\sum_{j=0}^{n-1}\left(\begin{array}{c}n-1 \\ j\end{array}\right)(-1)^{j} p^{k j}$, and simplifying the resulting geometric sum, we conclude that

$$
\mathrm{E}\left[D_{n}\right]=\frac{1}{q}-\sum_{j=0}^{n-1}\left(\begin{array}{c}
n-1 \\
j
\end{array}\right) \frac{(-1)^{j}}{1-p^{j+1}} .
$$

The alternating sum in the mean can be handled by Rice's integral method [4]. The fundamental idea is to recognize that such an alternating sum is exactly the same as

$$
-\frac{1}{2 \pi \mathrm{i}} \oint_{C_{1}} \frac{\beta(n,-z)}{1-p^{z+1}} \mathrm{~d} z
$$

where $\beta(\cdot, \cdot)$ is the beta function, and the line integral is taken over a closed contour $C_{1}$ 
surrounding the integers $0,1, \ldots, n-1$ and no other integers. For instance, $C_{1}$ can be the contour consisting of a rectangle connecting the four corners at $-\frac{1}{2} \pm \mathrm{i}$ and $n-\frac{1}{2} \pm \mathrm{i}$. The link is that the integrand has simple poles at the integer points $0,1, \ldots, n-1$, and their residues coincide with the negative of the summands. The integral is then evaluated via the residues of the poles outside $C_{1}$ and a small error. This is done by deforming $C_{1}$ into a larger contour $C_{2}(a, M)$, say a rectangle connecting the four corners at $-a \pm \pi(2 M+1) \mathrm{i}$ and $n+b \pm \pi(2 M+1) \mathrm{i}$ for $a, b>1$ and a large positive integer $M$. The two integrals over $C_{1}$ and $C_{2}(a, M)$ differ by the residues of the poles enclaved between the two contours. Outside $C_{1}$, there are simple poles at $z_{k}=-1+2 \pi k \mathrm{i} / \ln p$ for $k=0, \pm 1, \pm 2, \ldots$. Thus, as we let $M \rightarrow \infty$, the rectangle $C_{2}(a, M)$ grows to encompass all the poles outside $C_{1}$, and

$$
-\frac{1}{2 \pi \mathrm{i}} \oint_{C_{1}} \frac{\beta(n,-z)}{1-p^{z+1}} \mathrm{~d} z=-\frac{1}{2 \pi \mathrm{i}} \lim _{M \rightarrow \infty} \oint_{C_{2}(a, M)} \frac{\beta(n,-z)}{1-p^{z+1}} \mathrm{~d} z+\sum_{k=-\infty}^{\infty} \operatorname{Res}_{z=z_{k}} \frac{\beta(n,-z)}{1-p^{z+1}} .
$$

By Stirling's approximation to the gamma function, we have the residue calculation

$$
\operatorname{Res}_{z=z_{k}} \frac{\beta(n,-z)}{1-p^{z+1}}=-\frac{\Gamma(n) \Gamma\left(-z_{k}\right)}{\Gamma\left(n-z_{k}\right) \ln p}=-\frac{n^{-1+2 \pi k \mathrm{i} / \ln p}}{\ln p} \Gamma\left(1-\frac{2 \pi k \mathrm{i}}{\ln p}\right)\left(1+O\left(\frac{1}{n}\right)\right) .
$$

As $M \rightarrow \infty$, the integral over the limiting contour $\lim _{M \rightarrow \infty} C_{2}(a, M)$ gives a correction error of the order $n^{-a}$. As we already took only one term in Stirling's approximation, it does not help to take $a$ much greater than 1 . Let us take $a=2$ and obtain

$$
\mathrm{E}\left[D_{n}\right]=\frac{1}{q}+\left(\frac{1}{\ln p} \sum_{k=-\infty}^{\infty} \Gamma\left(1-\frac{2 \pi \mathrm{i} k}{\ln p}\right) \mathrm{e}^{-2 \pi \mathrm{i} k \log _{1 / p} n}\right) \frac{1}{n}+O\left(\frac{1}{n^{2}}\right) .
$$

We can improve the overall error by taking more terms in Stirling's approximation, and choosing larger $a$.

The variance follows from similar developments, which we only sketch. The exact second factorial moment is

$$
\mathrm{E}\left[D_{n}\left(D_{n}-1\right)\right]=\sum_{k=0}^{\infty} k(k-1) \mathrm{P}\left(D_{n}=k\right)=2 \sum_{j=1}^{n-1}\left(\begin{array}{c}
n-1 \\
j
\end{array}\right) \frac{(-1)^{j+1} p^{j+1}}{\left(1-p^{j+1}\right)^{2}} .
$$

We manipulate the formula for the exact probability to be in the form of an alternating sum, which we then handle via Rice's integral method. The result follows after a residue calculation.

\section{Total number of coin flips}

Let $X_{n}$ be the total number of coin flips until the contest comes to a halt. Under serialized conditions, $X_{n}$ is a measure of the speed of the algorithm. Let $W_{n}$ be the number of candidate winners who move to the next round. The number $W_{n}$ is a binomially distributed random variable counting the number of successes in $n$ independent, identically distributed trials with rate of success $p$ per trial. For $n \geq 2$, we have

$$
X_{n} \stackrel{\mathrm{D}}{=} n+X_{W_{n}}
$$

the initial conditions are $X_{1}=X_{0}=0$. Note that

$$
X_{n}=D_{n, 1}+D_{n, 2}+\cdots+D_{n, n} .
$$


The random durations are identically distributed but are dependent (in fact they are exchangeable). This adds complexity to the study of the distribution of $X_{n}$. However, this does not introduce an added difficulty for calculation of the mean:

$$
\mathrm{E}\left[X_{n}\right]=\sum_{j=1}^{n} \mathrm{E}\left[D_{n, j}\right]=n \mathrm{E}\left[D_{n}\right]=\frac{n}{q}+\frac{1}{\ln p} \sum_{k=-\infty}^{\infty} \Gamma\left(1-\frac{2 \pi \mathrm{i} k}{\ln p}\right) \mathrm{e}^{-2 \pi \mathrm{i} k \log _{1 / p} n}+O\left(\frac{1}{n}\right) .
$$

In view of the dependence, higher moments are harder to compute directly from recurrences derived from (2). However, the form of (1) is amenable to convergence methods in metric spaces, as simple limits can be guessed. The binomial distribution of $W_{n}$ can be approximated by a normal distribution with mean $n p$ and variance $n p q$. (In what follows a normal random variate with mean $\mu$ and variance $\sigma^{2}$ will be denoted by $\mathcal{N}\left(\mu, \sigma^{2}\right)$.) This suggests that $\sqrt{n}$ is the right scale factor for limits to appear on the right-hand side. Centering and scaling (1), we obtain

$$
X_{n}^{*}:=\frac{X_{n}-n / q}{\sqrt{n}} \stackrel{\mathrm{D}}{=} \frac{X_{W_{n}}-W_{n} / q}{\sqrt{W_{n}}} \sqrt{\frac{W_{n}}{n}}+\frac{n-n / q+W_{n} / q}{\sqrt{n}} .
$$

Thus, in normalized form we have the distributional equation

$$
X_{n}^{*} \stackrel{\mathrm{D}}{=} X_{W_{n}}^{*} \sqrt{\frac{W_{n}}{n}}+\frac{n-n / q+W_{n} / q}{\sqrt{n}} .
$$

Now, if $X_{n}^{*} \stackrel{\mathrm{D}}{\rightarrow} X^{*}$, so will $X_{W_{n}}^{*}$ because $W_{n} \stackrel{\text { a.s. }}{\rightarrow} \infty$. Also, $\sqrt{W_{n} / n} \stackrel{\mathrm{P}}{\rightarrow} \sqrt{p}$. It would then follow from Slutsky's theorem (see [6, p. 147]) that the combination $X_{W_{n}}^{*} \sqrt{W_{n} / n}$ converges in distribution to $X^{*} \sqrt{p}$. Furthermore, $\left(n-n / q+W_{n} / q\right) / \sqrt{n} \stackrel{\mathrm{D}}{\rightarrow} Z \sqrt{p / q}$, where $Z \stackrel{\mathrm{D}}{=} \mathcal{N}(0,1)$. Hence, if $X_{n}^{*}$ has a limit, we would expect it to satisfy the distributional equation

$$
X^{*} \stackrel{\mathrm{D}}{=} \sqrt{p} X^{*}+\sqrt{\frac{p}{q}} Z .
$$

(Technically, $X^{*}$ turns out to be the fixed point of the contraction given implicitly by the distributional equation (3). The contraction method was introduced by Rösler [14] to analyze the Quick Sort algorithm. A broad theory is developed in [10], and [15] provides a survey.) To rigorously prove all this, it suffices to show that the second-order Wasserstein distance $d_{2}\left(F_{n}^{*}, F^{*}\right)$ converges to 0 , as $n \rightarrow \infty$, where $F_{n}^{*}$ is the distribution function of $X_{n}^{*}$ and $F^{*}$ is the distribution function of $X^{*}$. The Wasserstein distance of order 2 between two distribution functions $F_{n}^{*}$ and $F^{*}$ is defined by

$$
d_{2}\left(F_{n}^{*}, F^{*}\right):=\inf _{V_{n}^{*}, V^{*}}\left\{\left\|W_{n}^{*}-W\right\|_{2}: W_{n}^{*} \stackrel{\mathrm{D}}{=} V_{n}^{*}, W \stackrel{\mathrm{D}}{=} V^{*}\right\},
$$

where $V_{n}^{*}$ is of distribution $F_{n}^{*}, V^{*}$ is of distribution $F^{*}$, and $\|\cdot\|_{2}$ is the $\mathcal{L}_{2}$ norm. Thanks to recent advances in the contraction method, such as those in [9], we can prove in a fairly mechanical way that $X_{n}^{*} \stackrel{\mathrm{D}}{\rightarrow} X^{*}$. Such convergence implies convergence in the first two moments too [1, p. 254]. The next lemma characterizes the limit.

Lemma 1. Let $Z$ be a standard normal random variable. If a random variable $X^{*}$ is independent from $Z$ and satisfies the distributional equation

$$
X^{*} \stackrel{\mathrm{D}}{=} \sqrt{p} X^{*}+\sqrt{\frac{p}{q}} Z
$$

then $X^{*}$ has the distribution of $\mathcal{N}\left(0, p / q^{2}\right)$. 
Proof. Let $\eta_{X}^{*}(t)$ be the characteristic function of $X^{*}$. Both sides of the distributional equation have the same characteristic function:

$$
\begin{aligned}
\eta_{X^{*}}(t) & =\eta_{X^{*}}\left(p^{1 / 2} t\right) \exp \left(-\frac{p t^{2}}{2 q}\right) \\
& =\eta_{X^{*}}\left(p^{2 / 2} t\right) \exp \left(-\frac{p t^{2}}{2 q}-\frac{p^{2} t^{2}}{2 q}\right) \\
& =\cdots \\
& =\eta_{X^{*}}\left(p^{k / 2} t\right) \exp \left(-\frac{p t^{2}}{2 q}\left(1+p+p^{2}+\cdots+p^{k-1}\right)\right) .
\end{aligned}
$$

Take the limit, as $k \rightarrow \infty$. By dominated convergence, the function $\eta_{X^{*}}\left(p^{k / 2} t\right)$ has the limit $\eta_{X^{*}}(0)=1$. Hence,

$$
\eta_{X^{*}}(t)=\exp \left(-\frac{p t^{2}}{2 q^{2}}\right),
$$

and the right-hand side is the characteristic function of $\mathcal{N}\left(0, p / q^{2}\right)$.

The convergence of $X_{n}^{*}$ to a limit $X^{*}$ satisfying the distributional equation in Lemma 1 is demonstrated in [9]; see Remark 1 below. This convergence, together with the characterization in Lemma 1, establishes the following result.

Theorem 2. As $n \rightarrow \infty$,

$$
\frac{X_{n}-n / q}{\sqrt{n}} \stackrel{\mathrm{D}}{\rightarrow} \mathcal{N}\left(0, \frac{p}{q^{2}}\right) .
$$

Remark 1. This development is quite similar to the general result in Theorem 6.1 and Corollary 6.2 of [9] which is an application to the size of a random skip list. The construction algorithm of skip lists [2], [11], [13] is a bit different from the leader election algorithm presented in this paper, but gives the same type of stochastic recurrence, only differing in initial conditions [9].

\section{Acknowledgements}

The first author wishes to thank the Columbian School of Arts and Sciences at The George Washington University and the Fields-MITACS Summer School in Applied Probability for grants that made presentation and completion of this work possible. The authors thank Robert Smythe for technical advice and an anonymous reviewer for input that allowed a more concise presentation.

\section{References}

[1] Barbour, A. D., Holst, L. And Janson, S. (1992). Poisson Approximation. Clarendon Press, Oxford.

[2] Devroye, L. (1992). A limit theory for random skip lists. Ann. Appl. Prob. 2, 597-609.

[3] Fill, J. A., Mahmoud, H. M. And SzPankowski, W. (1996). On the distribution for the duration of a randomized leader election algorithm. Ann. Appl. Prob. 6, 1260-1283.

[4] Flajolet, P. and Sedgewick, R. (1995). Mellin transforms and asymptotics: finite differences and Rice's integrals. Theoret. Comput. Sci. 144, 101-124.

[5] JANSON, S. AND SzPAnKowsKi, W. (1997). Analysis of an asymmetric leader election algorithm. Electron. J. Combinatorics 4, R17, 16pp.

[6] Karr, A. F. (1993). Probability. Springer, New York.

[7] Louchard, G. And Prodinger, H. (2008). Advancing in the presence of a demon. Math. Slovaca 58, $263-276$. 
[8] Louchard, G., Prodinger, H. and Ward, M. D. (2011). Number of survivors in the presence of a demon. To appear in Periodica Mathematica Hungarica.

[9] NeInInger, R. (2005). Recursive random variables with subgaussian distributions. Statist. Decisions 23, 131146.

[10] NeININGer, R. AND RÜschendorf, L. (2004). A general limit theorem for recursive algorithms and combinatorial structures. Ann. Appl. Prob. 14, 378-418.

[11] Papadakis, T., Munroe, J. I. and Poblete, P. V. (1990). Analysis of the expected search cost in skip lists. In SWAT 90 (Bergen, 1990; Lecture Notes Comput. Sci. 447), Springer, Berlin, pp. 160-172.

[12] Prodinger, H. (1993). How to select a loser. Discrete Math. 120, 149-159.

[13] Pugh, W. (1989). Skip lists: a probabilistic alternative to balanced trees. In Algorithms and Data Structures (Ottawa, ON, 1989; Lecture Notes Comput. Sci. 382), Springer, Berlin, pp. 437-449.

[14] Rösler, U. (1991). A limit theorem for “Quicksort”. RAIRO Inf. Théor. Appl. 25, 85-100.

[15] Rösler, U. AND RÜschendoRf, L. (2001). The contraction method for recursive algorithms. Algorithmica 29, 3-33. 